\title{
Corela
}

Cognition, représentation, langage

16-1 | 2018

Vol. $16, \mathrm{n}^{\circ} 1$

\section{La Grammaire à l'épreuve de la langue et de la métalangue}

Le cas de l'enseignement de l'anglais langue seconde.

\section{Clotilde Castagné-Véziès}

\section{OpenEdition}

\section{Journals}

Édition électronique

URL : http://journals.openedition.org/corela/5394

DOI : $10.4000 /$ corela.5394

ISSN : 1638-573X

Éditeur

Cercle linguistique du Centre et de I'Ouest - CerLICO

\section{Référence électronique}

Clotilde Castagné-Véziès, « La Grammaire à l'épreuve de la langue et de la métalangue », Corela [En ligne], 16-1 | 2018, mis en ligne le 28 juin 2018, consulté le 30 avril 2019. URL : http://

journals.openedition.org/corela/5394 ; DOI : 10.4000/corela.5394

Ce document a été généré automatiquement le 30 avril 2019

\section{c) (1)(2)}

Corela - cognition, représentation, langage est mis à disposition selon les termes de la licence Creative Commons Attribution - Pas d'Utilisation Commerciale - Partage dans les Mêmes Conditions 4.0 International. 


\title{
La Grammaire à l'épreuve de la langue et de la métalangue
}

Le cas de l'enseignement de l'anglais langue seconde ${ }^{1}$.

\author{
Clotilde Castagné-Véziès
}

\section{Introduction : un double paradoxe}

1 'Linguistics is harder than maths.' : ce commentaire trouvé dans la copie (par ailleurs blanche) d'une étudiante angliciste de L3 (LLCER), pour le devoir final de «Syntax and Grammar ", exprime à sa façon le ressenti de beaucoup d'étudiants qui doivent valider un enseignement obligatoire de grammaire anglaise dans le cadre de leurs études d'anglais. S'ils ne le verbalisent pas ainsi, une même forme d'inquiétude est également palpable chaque année chez les collègues qui se lancent dans la préparation du concours de l'Agrégation interne d'anglais : mais le public est forcément différent et très vite, le cours de « Grammaire linguistique » pour l'analyse des « Faits de Langue », à l'oral du concours, suscite énormément de questions, comme si soudain, l'occasion était enfin donnée de prendre un nouveau départ avec la grammaire, selon une approche linguistique de la langue.

2 La comparaison que fait l'étudiante, entre la linguistique -- pour désigner la grammaire linguistique -- et la mathématique ${ }^{2}$, est parlante par le rapprochement qu'elle établit entre les sciences humaines ${ }^{3}$ et les sciences exactes, touchant là à un premier paradoxe général : la grammaire, perçue comme une science exacte avec son mécanisme de règles grammaticales, apparait pour beaucoup comme une discipline exigeante et difficile, mal maitrisée ${ }^{4}$, et par là souvent effrayante et repoussante ${ }^{5}$; mais dans le même temps, elle est prisée par d'autres comme un exercice intellectuel de formation de l'esprit, elle devient alors sélective ${ }^{6}$ - chaque époque a ses « forts en thème » -- et va pouvoir jouer un rôle rassurant pour les parents, les enseignants et certains cadres dirigeants car elle pose des bases. On entendra souvent déplorer qu'« on ne fait plus assez de grammaire »..., ou bien on y verra un moyen de redresser la situation. ${ }^{7}$ 
Ce 1er paradoxe se double, pour la grammaire anglaise, d'un 2ème paradoxe : en effet, comparé avec autres langues à morphologie plus complexe (car elles ont notamment des déclinaisons, des conjugaisons, comme l'allemand ou le polonais par exemple), l'anglais est considéré par beaucoup de francophones comme une langue "facile " à apprendre, car sans grammaire ou presque! Ceci était déjà constaté par Yaguello (1988:135), et le résultat de l'enquête menée auprès d'étudiants de L1 à l'Université catholique de l'Ouest, et publiée en 2008 par Le Lievre, dans sa thèse de doctorat ${ }^{9}$, le corrobore. Des entretiens sont organisés, et l'une des questions posées est la suivante: "Maintenant, en ce qui concerne la facilité ou la difficulté de l'anglais, qu'est-ce que vous en diriez? Qu'est-ce que vous pensez de l'anglais? Vous pensez que c'est facile? Que c'est difficile? Ou qu'estce qui vous semble facile ou vous semble difficile?». «70\% des étudiants spécialistes ${ }^{10}$ déclarent que l'anglais est une langue facile.» (Le Lievre 2008: 255) L'auteur souligne que: "C'est un résultat surprenant puisqu'habituellement, plus l'on avance dans une langue, plus l'on envisage son apprentissage comme se complexifiant.» (Le Lievre 2008: 256). Par ailleurs, « $40 \%$ des étudiants non-spécialistes ${ }^{11}$ ne considèrent pas l'anglais comme une langue difficile », ce qui représente un fort pourcentage pour des étudiants qui étudient un tout autre domaine que les langues. Le Lievre affine ces résultats en montrant que, pour les anglicistes, si la compréhension est facile, l'expression orale l'est moins, et c'est la grammaire qui est considérée comme le plus difficile : « l'anglais devient difficile pour les spécialistes parce qu'ils sont avancés dans la langue. De plus, souvenonsnous que la maitrise de la grammaire en France est considérée comme très importante, elle est, bien souvent, encore vécue comme un indice de bonne réussite scolaire » (Le Lievre 2008: 258). Cela nous ramène au $1^{\mathrm{er}}$ paradoxe. Dès lors, faut-il vraiment s'intéresser à la grammaire de l'anglais ? peut-on et doit-on l'enseigner?

\section{Linguistique et grammaire : un enjeu stimulant}

Nous voyons au travers du premier paradoxe qu'il convient de distinguer deux plans, celui de la recherche scientifique et celui des relations sociales. La linguistique, comme science, a pour objet l'étude du langage, et cette étude ne peut se faire qu'à travers la diversité des langues du monde. Par grammaire «linguistique ", il faut entendre «les divers modèles (au sens scientifique) qui entendent représenter la structure d'une langue. » (Mainguenau, 2013: 34). Les relations sociales entre êtres humains nécessitent quant à elles une maîtrise de la langue première (voire de plusieurs langues premières, d'une langue seconde ou de langues étrangères) et font de la grammaire un enjeu incontournable : il faut maîtriser la structure de la langue pour communiquer, c'est-àdire, comprendre et se faire comprendre. Ce qui a une retombée immédiate sur l'enseignement: si l'école est le lieu des apprentissages, elle va réserver à la grammaire un créneau dans l'emploi du temps, au même titre que l'histoire ou les mathématiques, elle va mettre en pratique cet apprentissage et « faire de la grammaire ${ }^{12}$

5 La grammaire linguistique, en tant que science, permet d'observer les phénomènes de la langue et elle fournit des outils de description et d'explication de la langue. Cependant, il faut «se prémunir contre les redoutables pièges de la pseudo-rigueur. [La grammaire] comme toutes les sciences humaines, (...) est faite de repères forts et de certitudes, mais aussi de zones plus fluctuantes pour lesquelles la réflexion et la nuance sont de mise. » (Léon 2008/2014 : 99). Prenons l'exemple de l'emploi des verbes lexicaux DO et MAKE en anglais, délicat pour les francophones qui, eux, n'utilisent souvent qu'un 
seul verbe, «faire». On explique parfois que MAKE serait plus concret et on proposera souvent de le traduire par «fabriquer » en français, tandis que DO le serait moins. Mais alors, pourquoi dit-on en anglais «make a cake » et « do the dishes », qui renvoient tous deux à une activité «concrète » du monde extra-linguistique? et par ailleurs «make war », mais «do justice » là où, combinés à deux noms avec le déterminant article "zéro ", ils renvoient tous deux à une idée plus abstraite, la guerre, la justice? Nous voyons ici qu'il faut affiner le raisonnement pour pouvoir rendre compte de l'emploi des verbes lexicaux DO et $\mathrm{MAKE}^{13}$. De la même façon, nous pouvons nous demander pourquoi l'anglais va dire: "you can take the train to Birmingham ", ou " you can take the bus to Birmingham ", mais pas " you can take *the cab to Birmingham ", et difficilement " 'you can take the plane to Birmingham »; l'anglais préfèrera : " you can fly to Birmingham », ce qui amène à s'interroger alors sur l'emploi des déterminants articles ${ }^{14}$. Faire prendre conscience de ces différences, et tenter d'en rendre compte en situant toujours la langue dans son contexte pragmatique, est tout l'enjeu de la grammaire linguistique.

\section{Les représentations de la grammaire: hypothèses}

6 La prise en compte de la pragmatique, et du matériau vivant et sans cesse changeant qu'est la langue, amène à considérer aussi la grammaire dans le cadre de l'interaction sociale. Les représentations sociales ${ }^{15}$, terme issu du domaine des sciences sociales, en particulier la psychologie sociale, sont pour Jodelet des «formes de connaissances socialement élaborées et partagées, ayant une visée pratique et concourant à la construction d'une réalité commune à un ensemble social » (Jodelet 1989 ; 2003 : 53). Il faut distinguer la notion de représentation de celles d'attitude et de stéréotype. "L'attitude est généralement définie comme une disposition à réagir de manière favorable ou non à une classe d'objet ", sur laquelle l'individu possède un «stock de croyances ", qui peuvent reposer sur des informations objectives ou des stéréotypes. Le stéréotype est à comprendre comme "une forme spécifique de verbalisation d'attitudes ", qui affiche «les perceptions identitaires et la cohésion des groupes », en permettant de les situer les uns par rapport aux autres. (Castelloti-Moore, 2002: 7-9). Enfin, il faut retenir trois critères propres aux représentations sociales et liés entre eux:«leur élaboration dans et par la communication, la (re)construction du réel et la maîtrise de l'environnement par son organisation ».

7 Outil d'analyse en psychologie cognitive, depuis une trentaine d'années, la notion de représentation est prégnante également dans les études sur les langues, que ce soit dans le domaine de la sociolinguistique, l'acquisition du langage, ou la didactique des langues. Les politiques linguistiques éducatives en Europe, avec le CERCL (Cadre Européen Commun de Référence pour les Langues), reconnaissent le rôle joué par les représentations sociales dans la construction de soi, le rapport aux autres et la construction des connaissances, au travers de l'apprentissage et de l'enseignement des langues.

8 Pour aller plus loin, en appliquant le concept au domaine de la grammaire, nous défendons l'hypothèse que les représentations de la grammaire jouent un rôle très important dans l'apprentissage et l'enseignement de la grammaire; qu'elles influencent les attitudes envers la grammaire et l'intérêt que les apprenants lui portent. Ainsi Hubert (2016) a fait s'exprimer des étudiants sur leur vécu scolaire. Il montre qu' « un travail sur les relations à la grammaire qu'on a développées comme élève semble particulièrement 
nécessaire pour ne pas reproduire (...) un enseignement qui ne «fonctionne " pas pour une majorité des individus. La prise de conscience que permet l'expression des représentations sur la séance de grammaire donne la possibilité d'envisager d'autres rapports à l'apprentissage.» (Hubert 2016: 120). Si une représentation est toujours représentation de quelqu'un et de quelque chose (Moscovici 1961: 76, 96), la représentation de la grammaire va jouer un rôle essentiel dans l'acquisition de la connaissance et par là, de la maîtrise de la langue, par l'apprenant. On sait en effet que les représentations sociales peuvent fortifier ou ralentir les processus d'apprentissage (Castelloti-Moore, $2002: 10$ ).

9 Py identifie un modèle «fondé par l'opposition entre représentations de référence et représentation en usage ", où la valeur de référence, repère commun à tous, est stable (du côté de la préconstruction) tandis que la valeur individuelle est évolutive, élaborée au fil de l'interaction (du côté de la co-construction). (Py 2000: 12 et 14). Si on applique ce modèle à la grammaire, les représentations de référence, très négatives chez la plupart des apprenants (Crystal 20046), et les enseignants (Hubert 2016:117 ${ }^{17}$ ), et préalables à l'apprentissage ou à l'enseignement, créent une forme d'anticipation angoissée ou désillusionnée qu'il va falloir dépasser pour permettre à l'apprenant, à l'enseignant, voire plus largement à tout un chacun, de re-présenter - re-créer, co-construire -- la réalité grammaticale. Chaque individu, s'il est à la fois tributaire des représentations de référence - le plus souvent négatives, en ce qui concerne la grammaire, comme nous venons de le voir -- peut choisir individuellement, et dans l'interaction avec le groupe, la façon dont il répond à cette situation. Certes, la sédimentation est forte, et il est donc urgent d'agir au niveau des représentations en usage de la grammaire, pour modifier les attitudes et les stéréotypes, et accroître ainsi la maîtrise grammaticale..$^{18}$ Nous pouvons, pour cela, nous intéresser à la «réception du discours grammatical» et travailler par exemple à partir des "représentations naïves" que livre "l'ordinaire des mots » (Lapaire, 2016 : § 42). Nous y revenons dans la partie 7.

10 Aux représentations de la grammaire, prises entre le complexe de mal maitriser la grammaire, cette grammaire aux règles arbitraires ${ }^{19}$, redoutables et redoutées ${ }^{20}$, et le pouvoir sur la langue et sur autrui par la maitrise de règles grammaticales idéalisées, s'ajoute l'obstacle de la métalangue grammaticale que l'on considère à présent.

\section{L'obstacle de la métalangue grammaticale}

11 Dans le cadre de la préparation au concours de l'Agrégation interne d'anglais, des collègues agrégatifs nous posent un jour par email les questions suivantes ${ }^{21}$ :

12 «Que signifie l'expression «insertion pragmatique "?, Qu'est-ce que la «diathèse » (passive) ? Quelle est la différence entre «thématisation » et " topicalisation», s'il y en a une? Que sont la parataxe et l'hypotaxe? Qu'est-ce qu'une subordonnée enchâssée? Existe-t-il des semi modaux? Si non, que sont "Ought to / Need to / Want to"? Si oui, comment fonctionnent-ils ? Quelle est la différence entre contrôle et montée ?»

13 A ces questions, nous pourrions rajouter celles des étudiants anglicistes en cursus de Licence ou de Master : «Qu'est-ce que c'est un prédicat? un déictique? une anaphore? un quantifieur? quand est-ce qu'il faut dire 'syntagme nominal'? ou 'groupe nominal' ? ça ne serait pas un 'adjectif possessif' ? est-ce que c'est un gérondif? une gérondive? on n'a pas bien compris ce que c'est que l'aspect...». 
14 La linguistique ayant pour objet l'étude de la langue, et la grammaire l'étude de son système, le problème se pose de décrire et expliquer le fonctionnement de la langue en utilisant la langue elle-même ${ }^{22}$ ! D'où ce besoin d'utiliser une sorte d'autre langue grammaticale - une "métalangue, « au-delà de " ${ }^{23}$ la langue qu'on étudie. Ce qu'explique bien Emile Benveniste $(1974,35)$ : «La langue de la grammaire, qui décrit l'usage des formes de la langue, est une métalangue : parler de substantif, d'adverbe, de voyelle, de consonne, c'est une métalangue. Tout le vocabulaire de la métalangue ne trouve application que dans la langue.». Pour enseigner la grammaire linguistique à des étudiants anglicistes, on utilise un lexique spécifique en anglais, ou en français -- une métalangue et son métalexique -- pour parler des structures de l'anglais. Les « critères d'exactitude et de précision » (Lapaire, 2016, § 60) qui dictent les choix métalinguistiques du chercheur, complexifient la compréhension des phénomènes grammaticaux, plus qu'ils ne les éclairent. Lapaire (2016) pose la question de la recevabilité du discours grammatical, souvent taxé par les étudiants de jargon. Il évoque notamment « l'abstraction et la désincarnation (apparente) de très nombreuses notions et catégories : " prétérition », « aoriste ", « aspect », " perfectivité », « diathèse », « télique », « procès », « jussif », « déontique », « syntagme », « clitique », « déterminant déictique »... » (Lapaire 2016: § 43). Abstraction paradoxalement nécessaire à la compréhension des concepts, mais peu compréhensible. Nous verrons dans la partie 7 comment Lapaire (2016) propose de dépasser ce paradoxe.

15 La question se pose alors de la difficulté de passer d'un discours savant et universitaire, à un discours " appliqué à l'enseignant du secondaire » (ou étudiant-enseignant stagiaire) qui, à son tour, l'appliquera à l'élève. Cette question s'inscrit plus largement dans le champ de la circulation des savoirs, telle que Chevallard (1985) la problématise. Il développe le concept de "transposition didactique», en l'appliquant au champ de la didactique des mathématiques, et propose un modèle pour analyser la distance entre le savoir savant (savoir à enseigner) et le savoir enseigné (objet d'enseignement). Nous revenons sur ces questions dans les parties 7 et 8 .

16 A la lumière des questions posées ci-dessus par les agrégatifs internes et les étudiants, il apparait clairement qu'une terminologie hétéroclite est un premier obstacle majeur à la compréhension et à l'enseignement de la grammaire. Il semble possible de le surmonter en choisissant d'utiliser une métalangue grammaticale claire, contre le risque de «jargonner », qui plus est de façon dogmatique ${ }^{24}$ (par exemple, il est intéressant de parler de 'déictique', mais cela n'empêche aucunement d'identifier aussi un démonstratif, selon la grammaire traditionnelle). La clarté25 de cette métalangue grammaticale suppose :

17 - la nécessité de situer un terme " savant » dans le contexte de son modèle contre le risque de démultiplier les étiquettes (par exemple, l'emploi de "groupe nominal/GN» ou «syntagme nominal/SN», ou encore l'emploi de «complémenteur», ou d' « enchâssement »)

18 - la nécessité de lier les changements terminologiques à l'historicité (pour comprendre pourquoi et comment ils se sont produits - Chartrand $2017: 219)$ contre l'arbitraire des dénominations (par exemple, expliquer pourquoi ce qu'on dénommait 'adjectif possessif' est maintenant appelé un 'déterminant possessif')

19 - la nécessité, surtout pour un angliciste, de prendre conscience que certains mots grammaticaux sont empruntés à l'anglais et traduits tels quels (par exemple «topicalisation ») contre l'utilisation de ces mêmes mots comme de simples synonymes 
d'autres termes grammaticaux utilisés en grammaire française (pour cet exemple, utiliser indistinctement «topicalisation » et «focalisation », qui désigne un phénomène quelque peu différent).

20 - la nécessité de bien maîtriser en premier lieu la terminologie traditionnelle, de comprendre l'étymologie des termes (par exemple, "hypotaxe » et "subordination", issus du grec et du latin) contre la volonté d'introduire toujours plus de nouveaux termes, sans raison justifiée (par exemple, on peut s'interroger sur ce qui justifie l'utilisation relativement récente des termes « proximal » et « distal ${ }^{26}$, empruntés à l'anatomie, pour l'analyse des déictiques).

21 - la nécessité, enfin, de poser le choix clair d'une seule langue employée comme métalangue, pour l'enseignement de la grammaire anglaise langue seconde, à savoir l'anglais, contre des pratiques qui mêlent la langue première et la langue seconde, voire privilégient le plus souvent le français. ${ }^{27}$ Le mélange de deux métalangues grammaticales rajoute souvent de la confusion dans l'emploi des termes, qui ne recouvrent pas toujours la même réalité. Or « il est essentiel, pour aborder une langue étrangère, de se dégager des catégories et de la structure de la langue maternelle.» (Yaguello, 1988: 73) Par exemple, parler de "gérondif » à propos de certaines formes en V-ING pose problème car cela ne correspond pas toujours à ce que la grammaire française désigne par «le gérondif $\aleph^{28}$. De même, le système verbal de l'anglais, qui se construit sur deux temps morphologiques, deux aspects verbaux (le progressif et le parfait) et la modalité (avec le système des auxiliaires modaux, prototypique de l'anglais) fonctionne différemment de celui du français. C'est pourquoi il ne gagne pas à être expliqué avec les outils du système verbal $\mathrm{du}$ français qui distingue des modes et des temps, avec des variations morphologiques sur chaque personne conjuguée. Ainsi parler de conjugaison, ou de « conditionnel » à propos de l'anglais, en transposant la métalangue grammaticale du français, rend l'analyse difficile. On voit d'ailleurs que le $2^{\text {ème }}$ paradoxe propre à l'anglais, évoqué au début, est directement alimenté par cette métalangue: le prisme des catégories de la grammaire latine fait en effet percevoir à tort l'anglais comme une langue "sans grammaire ", car la métalangue des conjugaisons et déclinaisons y trouve peu d'écho. ${ }^{29}$

22 L'emploi d'une métalangue grammaticale nécessaire à la construction du savoir grammatical pose donc expressément l'exigence de sa lisibilité, de sa cohérence et de sa justesse pour qu'elle ne soit pas un obstacle.

\section{Définir les contours de la grammaticalité ou le 3ème paradoxe?}

23 Une fois les représentations de la grammaire modifiées, et surmonté l'obstacle de la métalangue, quelle que soit la langue et sa structure, le véritable enjeu de l'enseignement de la grammaire est de définir les contours de la grammaticalité. Et la grammaticalité s'évalue à l'épreuve de la langue : langue écrite, et langue orale ; langue "standard » et variétés de langues; langue dans son emploi formel et langue dans son emploi discursif et pragmatique ; langue dans sa dimension énonciative, cognitive et sociolinguistique, dans sa dimension synchronique ou diachronique.

24 Mais alors, n'y aurait-il pas de repères fixes ? c'est là encore un autre paradoxe. L'anglais standard, que ce soit le Southern British English ${ }^{30}$, ou le General American, n'est qu'une des 
variétés ${ }^{31}$ de l'anglais, la "variété standard ", qui correspond à un anglais plus formel, souvent écrit. Quelles que soit les autres variétés, elles sont définies par un certain nombre de régularités que l'on peut observer et décrire, autrement dit des règles, qui forment la grammaire de la langue pour cette variété et dont la grammaire linguistique tente de rendre compte: «one thing that laypersons don't always realize is that every dialect of every language is equally rule-governed and systematic.»(Biber 2015) ${ }^{32}$. Etablir, reconnaître et enseigner un standard de la langue, n'est pas instaurer une suprématie de ce standard mais permettre, grâce à un certain nombre de règles grammaticales, de baliser l'usage et l'emploi de la langue et d'en comprendre un peu mieux le fonctionnement : pour construire le sens, éliminer de l'ambiguïé et permettre la communication $^{33}$ (comprendre et se faire comprendre). «What's important to realize is that there's no such thing as a 'sloppy' or 'lazy' dialect. Every dialect of every language has rules - not 'schoolroom' rules, like 'don't split your infinitives', but rather the sorts of rules that tell us that 'the cat slept' is a sentence of English, but 'slept cat the' isn't. These rules tell us what language is like rather than what it should be like. » (Biber 2012).

Très souvent, les locuteurs-apprenants s'arrêtent dans leur processus d'acquisition car ils pensent en savoir assez pour « fonctionner ». Or, dans l'acquisition d'une langue seconde, sans règles, on atteint vite un palier. Une étude empirique est menée par Rossi (2017) avec des étudiants de L1 LEA, sur l'acquisition de la maîtrise de l'alternance du génitif en anglais, avec le complément prépositionnel ou le nom composé, là où le français ne possède qu'une seule forme synthétique, la construction prépositionnelle. Rossi montre que l'introduction progressive de règles aide le groupe d'apprenants de niveau bas, et qu'ils sont sensibles à la fréquence : plus l'étudiant a de règles, plus son niveau augmente, un apprentissage explicite est mis en place et les jugements d'acceptabilité sont plus confiants et plus justes. L'impact de la connaissance des règles est mesuré dans un examen final où les statistiques montrent que les étudiants qui connaissent le mieux les règles enregistrent un progrès presque deux fois supérieur à ceux qui les connaissent le moins. Le groupe de niveau plus avancé au départ, exposé à un «input» enrichi (des supports avec beaucoup de formes issues du corpus $\mathrm{GloWbE}^{34}$, qui ressortent en gras) et non pas aux règles, progresse moins et on observe très vite un nivellement chez ces apprenants.

Les règles grammaticales sont donc nécessaires pour pouvoir progresser, même lorsque l'objectif se veut une "lingua franca » ou langue véhiculaire (Global English) : en atteste, pour les francophones, l'expérience du Globish, qui s'il met en avant la maitrise du lexique - les « 1500 mots pour communiquer facilement dans le monde entier » -- n'en souligne pas moins l'importance de la grammaire (Nerrière, $2015: 94)$ : « La grammaire, pour finir, est déterminante: plus elle est correcte, meilleur est le résultat. L'effort ne doit pas s'arrêter à mi-chemin. Une syntaxe pertinente et complète améliorera vos chances d'être compris (...)» (avec des non-natifs ou des anglophones natifs). «Cela est absolu dans la langue parlée, et encore plus en langue écrite. »

La linguistique de corpus, auquel l'informatique donne désormais accès, offre quant à elle un outil très intéressant pour tester statistiquement des hypothèses, au moyen d'énoncés attestés regroupés en corpus, tels le BNC (British National Corpus), le CoCA (Corpus of Contemporary American English), ou le GloWbE mentionné ci-dessus, pour n'en citer que quelques-uns. Mais pour l'apprenant (élève, étudiant), et l'enseignant, elle est aussi un puits sans fond ...; car à tout moment, une "règle", qu'elle soit acquise par un enseignement explicite ou implicite, est susceptible d'être démentie par une nouvelle 
enquête et un nouvel algorithme. De la même façon, grâce à internet, l'exposition à l'anglais oral des séries suivies assidument par les apprenants permet une exposition pseudo-directe à la langue, qui vient illustrer des emplois parfois contradictoires de la langue.

Or une langue, par nature, change et évolue tout le temps. C'est un matériau vivant. Faute de quoi, elle devient une langue « morte ». On le voit tout particulièrement dans la façon dont les jeunes générations se réapproprient en permanence la langue. Elles qui manifestent souvent un rejet de la grammaire, assimilée à la pratique écrite de la langue, sont pourtant les premières à faire jouer à l'oral les structures de leur langue.

Comment est-il alors possible de définir les contours de la grammaticalité?

\section{6. «Construire du déformable ${ }^{35}$}

Il est possible de définir les contours de la grammaticalité, en posant des repères stables ${ }^{36}$, explicités dans une métalangue claire et définis précisément, selon un modèle donné et des règles ou principes (à défaut de théorie) ${ }^{37}$. C'est parce qu'il y cette stabilité de départ qu'on pourra ensuite accepter les variations dans la communication, que nous venons d'évoquer : « la langue est un système virtuel qui ne peut être appréhendé qu'à travers le discours et qui permet la compréhension mutuelle entre les utilisateurs. Sa mise en œuvre (...) implique nécessairement des variations plus ou moins importantes. Il ne fournit pas des phrases toutes faites, mais seulement les règles qui permettent de les construire. Ces règles peuvent être flexibles dans la mesure où elles n'altèrent pas la stabilité du système. (Larroque, 2012 : 248). Variations à l'intérieur du standard, et entre le standard et le moins ou non-standard. Ce qui fait intervenir le concept d'énonciation car toute production linguistique est en lien avec la situation dans laquelle elle est énoncée. Et «les règles de bonne formation énonciative ne sont pas nécessairement identiques aux règles de bonne formation de phrase." (Culioli 1999: 119). Pour ne prendre qu'un seul exemple dans une perspective contrastive entre l'anglais et le français, la dislocation multiple n'est pas acceptable en anglais, à l'inverse du français où elle est fréquente : «*me, my mother, she thinks... (Gardelle, $2010: 13$ ).

L'idée d'une flexibilité des règles, à l'intérieur d'un système stable ou entre deux systèmes, amène à s'intéresser au rapport entre le type et l'occurrence, tel que Ballier (2004) le pose à propos de la phonologie de l'anglais, dans l'analyse du segmental. Pour faire saisir ce rapport, il prend l'exemple des cours de phonétique à l'université faits par des lecteurs anglophones «dont l'inventaire phonologique s'écarte notablement des documents qu'ils diffusent en cours ", ce qu'ils expriment sous forme d'une contradiction « je ne prononce pas ce que j'enseigne » (Ballier, 2004: §4). Le type correspond ici à la réalisation décrite, sous forme de transcription, dans un système phonologique donné comme variété de référence (le Standard British English par exemple), et l'occurrence, à la réalisation propre à un locuteur. On note souvent un décalage, un écart entre le type et l'occurrence: «Ce que, littéralement, on donne à entendre n'est pas rigoureusement

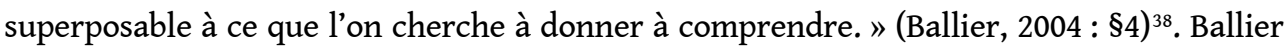
fait l'hypothèse qu'il faut pouvoir d'abord définir une norme ou standard, dans un cadre de référence, pour pouvoir ensuite s'en éloigner, ou saisir le rapport de l'occurrence au type : « la variété de référence vaut moins pour elle-même que pour les opérations de conversions qu'elle autorise dans l'intelligibilité mutuelle entre locuteurs de variétés 
différentes. ». Selon Ballier, on ne peut pas toujours « expliquer » ce rapport, qui peut même aller jusqu'à la rupture entre l'occurrence et le type.

Cette analyse nous semble transposable à la grammaire: de même que pour Ballier, en phonologie, «la question de la variété décrite (et par ailleurs de la variété parlée par l'enseignant) manifeste l'écart possible entre réalisations et transcriptions et pose de manière cruciale pour la linguistique anglaise le rapport entre le type et l'occurrence. ", de même en grammaire, on peut dire que la variété de l'anglais standard enseignée (et par ailleurs celle parlée et écrite par l'enseignant, selon son savoir acquis et disponible) manifeste l'écart possible entre cette norme didactique nécessaire (qui veut donner à comprendre le système de la langue anglaise) et la diversité des réalisations effectives de l'anglais (oral ou écrit, dépendant des usages et des codes sociaux), dans un rapport semblable entre le type et l'occurrence.

Cet écart par rapport à la règle peut cependant parfois être réduit à néant, et Walker (2010) montre à propos de l'emploi des modaux WILL/SHALL, et du prétérit après IF -- « If it were/If it was" -- comment l'influence de la «tradition prescriptiviste » de la grammaire, enseignée à des anglophones, conditionne ce rapport à tel point que l'occurrence s'identifie à la norme. Inversement, dans une expérimentation sur l'enseignement de la morphologie et la syntaxe des adjectifs avec des étudiants anglicistes en L2 à l'université, Hugou (2017) met en évidence un écart possible par rapport à la règle qui établit que le comparatif des adjectifs courts (1, voire pour certains 2 syllabes) se forme par suffixation du sous-morphème «-er " -- «nicer» -- tandis que celui d'un adjectif long ( 3 syllabes) se forme à l'aide de l'adverbe « more » suivi de la préposition (ou conjonction) «than" -- «more interesting ». Il relève par exemple dans son corpus constitué du script de 12 séries télévisées, l'occurrence : « more enchantinger » et montre comment, dans le contexte discursif donné, on peut légitimer cet emploi.

34 Comme nous invite y Culioli, construire du déformable semble être la voie pour dépasser l'arbitraire de règles perçues comme trop rigides, en proposant le repère stable des règles grammaticales du standard enseigné, en opérant des choix quant à cette variété grammaticale de référence, tout en jetant des ponts entre les diverses variétés d'anglais possibles et en procédant, dans l'interaction, à des ajustements par rapport à la forme schématique.

\section{Représentations, métalangue et grammaticalité : un triple fossé}

Une évolution des représentations de référence de la grammaire, par la prise en compte des représentations d'usage - notamment, à partir des représentations "naïves " du discours grammatical (Lapaire 2016) --, une clarification du métalexique et de la métalangue grammaticale employés, ainsi qu'une grammaticalité définie selon des repères stables mais aux contours déformables, devraient permettre de faire évoluer les rapports complexes entre :

36 - la culture grammaticale "scolaire », (« le lourd appareil grammatical» selon Weber, 2004: 10) des apprenants-étudiants -- avec ses incohérences et le poids de ses représentations -- et leur propre système grammatical qui leur permet de communiquer ;

- le savoir grammatical savant des universitaires (le discours métalinguistique savant et spécifique qu'ils utilisent) et le savoir grammatical appris et assimilé par les étudiants ; ce 
qui pose la question de la réception du discours grammatical, de son assimilation et de sa reconstruction;

- les savoirs grammaticaux et didactiques enseignés, utilisés par les enseignants métadiscours des enseignants, adaptés aux représentations qu'ils se font de la compréhension des apprenants-élèves (Trévise 1997) --, et leurs propres savoirs appris en tant qu'élèves, puis étudiants et enfin dans leur formation d'enseignants (Gauvin et al. $2017: 69-91)$

Selon Courtillon (2001:2), dans l'apprentissage d'une langue seconde, en collège et lycée, la grammaire est « un objet mythique d'apprentissage » et ne peut pas être un objectif en soi. La grammaire n'est pas seulement une connaissance déclarative (des règles qui décrivent ce qu'est la langue) mais une ressource saisie en contexte, qui pourra être réinvestie dans une nouvelle configuration discursive (pour comprendre le fonctionnement de la langue, des langues, et pour pouvoir «communiquer». (Jeanneret 2005). Dans le cadre de l'approche actionnelle, préconisée par les IO et le CECRL, cette re-contextualisation de la ressource grammaticale devra être mise en œuvre dans l'exécution de la tâche finale. Mais « pour être capable de repérer et de prélever des données grammaticales en contexte et en faire ainsi des ressources grammaticales, un apprenant doit avoir une certaine formation à l'analyse grammaticale. » Cela suppose la maîtrise «d'un savoir grammatical relevant d'un modèle de la langue apte à décrire ce type de liens ». (Jeanneret $2005: 20$ )

Cette maîtrise est nécessaire à tous les niveaux, celui de l'apprenant-élève, celui de l'apprenant-étudiant, et enfin celui de l'enseignant. "Les concepts savants de la grammaire linguistique sont-ils vraiment absorbés en bloc ou rejetés ? Ou ne subissent-ils pas plutôt des déformations? » s'interroge Lapaire (2016). L'enquête à laquelle il se livre auprès de sujets au sortir du lycée, puis en fin de premier semestre de la L1, auprès d'étudiants en première année d'études anglophones, lorsqu'ils ont suivi un cours de "grammaire théorique et appliquée », montre que «la métalangue instituée » est « plus ou moins maîtrisée », et cela se combine avec ce qu'on appelle souvent « des restes » -- de grammaire, en l'occurrence --, automatismes scolaires réducteurs, voire erronés, alors même que l'intuition sur la langue peut être très fine et juste. Autre enquête, autre public, celui d'enseignants-stagiaires du secondaire (Gauvin et al. 2017) : l'analyse de leur discours grammatical dans une séquence qui comprend un exercice sur l'identification des compléments verbaux (en français), met en évidence une grande fragilité dans la transposition didactique du savoir grammatical assimilé dans leur formation d'étudiants en Master enseignement, avec des inexactitudes, parfois des erreurs, et des raisonnements grammaticaux incomplets dans l'utilisation des manipulations syntaxiques. Il est d'autant plus important de prendre en compte ce constat sur la transposition du savoir savant, qui s'applique tant à l'apprentissage et l'enseignement du français langue maternelle qu'à ceux de l'anglais langue seconde ${ }^{39}$, que la formation en grammaire anglaise est dans la majorité des cas dispensée en français, ce qui croise les fragilités. Pour réagir, Lapaire (2016) suggère, pour l'anglais, la piste de la convergence entre l'acte de création (par l'enseignant) et l'acte de réception (par l'apprenant) (2016: §) : « Pour décrire de façon intelligible et accessible le fonctionnement de la langue, sans renoncer à l'exigence scientifique, il doit être possible de s'appuyer sur des notions, des situations et des opérations déjà maîtrisées, qui sont inscrites dans la langue et la culture de référence de la personne recevant l'explication grammaticale. " (Lapaire 2016, §43). Il expose une proposition concrète de convergence, dans le rapprochement que 
l'enseignant-grammairien peut établir entre l'expression des représentations naïves des étudiants d'une part et l'utilisation qu'il fait de la narration à visée grammaticale (NarraGram) et des phrases gestuelles (Kinegrams) d'autre part; le point de convergence étant la créativité ou génie de la langue pour décrire les faits grammaticaux. Il s'appuie là sur l'expérimentation déjà faite auprès d'un public de collégiens (Lapaire \& Masse 2006) et la présente à l'université «en mode décalé » (Lapaire, 2016: § 46) pour analyser le temps, l'aspect et la modalité, auprès du public d'étudiants anglicistes de L1. A l'issue de la présentation faite aux étudiants, de séquences narratives ou visuelles kinesthésiques, il évalue les scores de compréhension-mémorisation obtenus: très élevés pour le modal must et la mise en valeur de la force et la contre-force ( $89 \%)$, moins bons pour le modal should (55\%) sans doute car la représentation directionnelle du conseil est moins probante pour rendre compte de la force de persuasion de ce modal. Il cite des mesures effectuées à l'Université de Bordeaux Montaigne en 2014, trois mois après une unique présentation visuelle de captures d'écran, qui ont montré un taux de $94 \%$ de compréhension-mémorisation sur l'expression du prétérit et de l'aspect perfectif (V-ED, parfait). (Lapaire 2016: §52). Il faudrait certes pouvoir intégrer ce format à toute la structure de la séquence, mais en procédant par analogies visuelles-kinesthésiques, et en utilisant la métalangue des apprenants pour narrer la grammaire, cette approche montre une façon de dépasser l'obstacle de la métalangue et du métalexique. De façon plus large, on retiendra que « quelle que soit la démarche d'enseignement qu'il choisit de privilégier (transmissive, inductive, déductive, co-constructive, etc.), [le grammairien] doit s'interroger sur la manière dont les catégories savantes se réélaborent dans l'esprit de l'apprenant, à partir de connaissances résiduelles (ou acquis latents), des discours métalinguistiques auxquels on l'expose, d'activités métalinguistiques auxquelles on le convie. » (Lapaire 2016 : § 60).

\section{Au-delà des paradoxes}

41 On voit donc que le sentiment de non-maîtrise de la grammaire, et l'appréhension que soulève la simple évocation du mot « grammaire » chez les apprenants élèves, étudiants anglicistes en L, étudiants stagiaires du MEEF et parfois chez les enseignants de l'anglais langue seconde, sont liés à une imbrication de facteurs: linguistique (les acquis grammaticaux, sur fond de sédimentation, voire de fossilisation des représentations de référence), métalinguistique (la terminologie grammaticale, métalangue et métalexique) et didactique (la transposition ${ }^{40}$ ou « reconfiguration $»^{41}$ du savoir savant).

42 Au-delà des paradoxes soulignés, il semble possible de faire de la grammaire de l'anglais un outil efficace pour la maîtrise de la langue, écrite et orale, en travaillant conjointement sur ces facteurs. Il faudrait pour cela se concentrer sur 4 pôles essentiels :

43 - Définir avec les apprenants-étudiants une « variété standardisée » d'anglais, adaptée au niveau d'enseignement et la poser comme référence. Pour cela, élaborer ensemble des ressources grammaticales, et les règles qui les caractérisent, en partant de leurs représentations, et en tenant compte des repérages par rapport au monde, et des opérations de régulation et d'ajustement co-énonciatifs ${ }^{42}$.

44 - Aborder et enseigner la grammaire sous le triple axe de la syntaxe (construction et combinaison de formes), de la sémantique (sens d'une proposition et construction de la référence) et de la pragmatique (énonciation) ; et lier l'enseignement de la grammaire de l'écrit et de l'oral. 
45 iorer l'appropriation te la terminologie (voire parfois la terminologie elle-même) employer une terminologie claire et repérer les ambiguïtés entre la terminologie grammaticale de l'anglais et celle du français (métalexique); proposer alors, à tous les niveaux ${ }^{43}$, d'enseigner progressivement la grammaire anglaise en anglais, dans une métalangue langue accessible (outils linguistiques), qui doit être une aide à la grammaire.

46 - Former les futurs enseignants (en formation initiale du MEEF ${ }^{44}$ et dans une formation continue), à la maîtrise et l'emploi de cette métalangue (métalexique et outils linguistiques), afin qu'ils se sentent à l'aise pour pouvoir les utiliser eux-mêmes; pour cela, identifier dès le départ et régulièrement leurs savoirs acquis à partir de leurs savoirs enseignés. (Gauvin et al. 2017) ; en outre, situer les concepts grammaticaux et les règles dans leur contexte historique, dans le cadre d'une réflexion épistémologique qui permette d'assurer ensuite une cohérence interne aux contenus grammaticaux.

\section{Conclusion}

Les recherches actuelles en didactique de l'anglais, portant sur l'enseignement de la grammaire anglaise à des spécialistes, sont très peu nombreuses, et s'intéressent prioritairement aux pratiques enseignantes ${ }^{45}$. Dans une approche différente et complémentaire, cet argumentaire met en lumière l'importance de transformer les représentations de la grammaire et de la langue qu'ont les apprenants et les enseignants eux-mêmes. Il s'appuie sur l'anglais, en prenant le cas de l'enseignement de l'anglais langue seconde, parfois dans une perspective contrastive avec le français, et invite à réfléchir à la grammaire de la langue comme un système cohérent et construit qui donne accès au sens. L'étude insiste sur l'importance de bien accompagner l'appropriation des concepts grammaticaux (le sujet, le prédicat, les classes grammaticales,...), en travaillant sur la terminologie grammaticale et linguistique, y compris du point de vue épistémologique, et à la construction progressive et toujours reformulable des règles, avec leurs manques et parfois leurs incohérences, à l'image du matériau vivant qu'est la langue. Elle propose de mener l'analyse en articulant constamment un triple niveau, syntaxique, sémantique et pragmatique/énonciatif, et d'utiliser pour cela de bons outils, bien maîtrisés, qui emploient une métalangue claire et cohérente pour expliciter les phénomènes grammaticaux.

\section{BIBLIOGRAPHIE}

Ballier, N. (2004). « Quelques problèmes métalinguistiques en phonologie de l'anglais », Corela [En ligne], 2-1 | 2004, mis en ligne le 15 juin 2004, consulté le 21 février 2018. URL : http:// journals.openedition.org/corela/594 ; DOI : 10.4000/corela.594

Ban-Larrosa, J. Fontaine, P. (2015). New Password English $2^{\text {nde }}$, Anglais lycée $2^{\text {nde }}$ : Didier.

Benveniste, E. (1974). Problèmes de linguistique générale, tome 2. Paris :

Gallimard.

Corela, $16-1$ | 2018 
Birner, B. (2012). « Is English changing ? » Linguistic Society of America, https:// linguisticsociety.org./content/english-changing (consulté le 7 déc 2017)

Birner, B. (2015). Interview http://grammarist.com/nofront/22431/ (consulté le 7 déc 2017)

Boisson, C. (2005). « La terre, la barre de fer et le préfet de l'Isère : faits, lois / modèles, théories ». Anglophonia, 18/2005 : 7-27.

Chartrand, S.-G. (2017). « Le nécessaire rapport dialectique entre faire comprendre le fonctionnement de la langue et développer des compétences scripturales dans l'enseignement grammatical », 209-226, dans GAGNON, R. et BULEA-BRONCKART E., Former à l'enseignement de la grammaire. Villeneuve d'Ascq : Presses Universitaires du Septentrion.

Chevallard, Y. (1985). La Transposition didactique : du savoir savant au savoir enseigné. Grenoble : la Pensée Sauvage.

Courtillon, J. (2001/2). « La mise en oeuvre de la « grammaire du sens » dans l'approche communicative » Analyse de grammaires et de manuels, Ela. Études de linguistique appliquée, $\mathrm{n}$

- 122, 153-164.

Crystal, D. (2004). «In Word and Deed », TES Teacher, 30 April 2004, consulté le 28 mai 2018. URL : https://www.tes.com/news/word-and-deed-0

Culioli, A. (1986). “Stabilité et déformabilité en linguistique”, Etudes de Lettres, Langages et Connaissances. Université de Lausanne, repris dans T. 1 :127-134.

Culioli, A. (1999). Pour une linguistique de l'énonciation, t. 2. Paris : Ophrys.

Elalouf, M.-L. (2015). « Comment le nom passé est passé du langage courant à la terminologie grammaticale ?», dans Scolagram, [En ligne], $\mathrm{n}^{\circ} 1$ Enseigner/apprendre les oppositions aspectuelles. URL : http://scolagam.u-cergy.fr/

Gardelle, L. (2010). « Dislocations à gauche et antépositions de compléments en anglais : formes, fonctionnalités et insertion en discours », Discours [En ligne], 7 | 2010, mis en ligne le 20 décembre 2010, consulté le 30 septembre 2016. URL : http://discours.revues.org/7943 ; DOI : 10.4000/ discours.7943

Granger, G. (2001). "DO : trois fonctions, un schéma", Anaphores nominales et verbales, J-C.Souesme (ed), Cycnos, vol 18, $n^{\circ} 2$.

Hubert, B. (2016). « Formation des enseignants : le casse-tête de la grammaire. D'une recherche clinique dialogique à un nouveau dispositif de formation-recherche », Le français aujourd'hui 2016/1 ( $\left.{ }^{\circ} 192\right), 117-132$.

Hugou, V. (24 novembre 2017). « Pour une approche méta-grammaticale, enseigner le degré de l'adjectif en L2». Communication à la Journée d'étude : « Comment \& pourquoi enseigner la grammaire/linguistique anglaise aux spécialistes? », Institut du Monde Anglophone, Paris 3 (Maison de la Recherche, rue des Irlandais).

Jeanneret, T. (2005). « La place des ressources grammaticales dans l'organisation sociale des moyens langagiers ", 99-115, dans BRONCKART, J.-P. et al., Repenser l'enseignement des langues: comment identifier et exploiter les compétences. Villeneuve d'Ascq : Presses Universitaires du Septentrion.

Jodelet, D. (1989). Les Représentations sociales. Paris : PUF.

Gauvin, I. et al. (2017). « Les savoirs en grammaire et en didactique de la grammaire de futurs enseignants : une porte d'entrée pour réexaminer la formation à l'enseignement ", 69-96, dans 
Gagnon, R. et Bulea Bronckart E., Former à l'enseignement de la grammaire. Villeneuve d'Ascq : Presses Universitaires du Septentrion.

Lapaire, J.-R. (2006). La grammaire anglaise en mouvement. Paris : Hachette.

Lapaire, J.-R. (2006). Grammar in motion. D.V.D. En collaboration avec Jean Masse, chorégraphe. Paris : Hachette

Lapaire, J.-R. (2016). « Pour une théorie de la réception du discours grammatical », Anglophonia, 22, Construction du sens. Toulouse : P.U.M.

Larroque, P. (2012). « De la norme à la pratique... », dans L'ajustement dans la TOE d'Antoine Culioli, C. FILIPPI-DESWELLE (éd.), Collection linguistique Épilogos, 3. Rouen : Publications Électroniques de l'ERIAC, p. 235-250.

Le Lievre, F. (2008). « L'Anglais en France, une langue multiple : pratiques, représentations et postures d'apprentissage : une étude en contexte universitaire », Thèse de Doctorat en Lettres et Linguistique, http://www.theses.fr/2008TOUR2015.

Léon, R. (2014, $1^{\text {ère }}$ ed. 2008). Enseigner la grammaire et le vocabulaire à l'école. Paris: Hachette éducation.

Nerrière, J.-P. (2009). Don't speak English, parlez Globish, avec la liste révisée des 1500 mots pour communiquer facilement dans le monde entier. Paris : Eyrolles.

Pennec B. \& Delorme B. (2015). L'entraînement à la réflexion linguistique pour le concours du CAPES d'anglais. Toulouse : P.U.M.

Py, B. (2000). « Représentations sociales et discours. Questions épistémologiques

et méthodologiques », Tranel (Travaux neuchâtelois de linguistique) 32, 5-20.

Quivy, M. (2015). Réussir l'épreuve de traduction au CAPES d'anglais - Entraînement au commentaire linguistique. Paris, Ellipses.

Rossi, C. (24 novembre 2017). « Enseigner la grammaire anglaise en Licence : Pour une introduction progressive des règles. ». Communication à la Journée d'étude : « Comment \& pourquoi enseigner la grammaire/linguistique anglaise aux spécialistes ? », Institut du Monde Anglophone, Paris 3 (Maison de la Recherche, rue des Irlandais).

Rotgé, W. (2014). « Pourquoi la grammaire ? Pourquoi la grammaire anglaise à un public LANSAD ? ", Recherche et pratiques pédagogiques en langues de spécialité, vol. XXXIII N - 3 | 2014, mis en ligne le 15 octobre 2014, consulté le 28 mai 2018. URL : http:// journals.openedition.org/apliut/4872 ; DOI : 10.4000/apliut.4872

Saussure, F. de (2002). Écrits de linguistique générale. Paris : Gallimard.

Souesme, J.-C. (1989). "DO, deux valeurs, une fonction", Explorations en linguistique anglaise. Berne : Peter Lang.

Starck, Josette \& al. (2014). New Meeting Point Anglais 2de. Paris : Hatier.

Trévise, A. (1997). « Métalexique, métadiscours et interactions métalinguistiques », Linx, n 36, 1997. Métadiscours... Langues, Actes du colloque international du Groupe Recherche Jan Comenuis dans Linguistiques et Didactique des Langues (2-3 octobre 96) 41-54, généré le 16 octobre 2015. URL : http://www.persee.fr/doc/linx 0246-8743 1997 num 3611452 ; DOI : 10.3406/ linx.1997.1452

Truchot, C. (2005). «L'anglais comme "lingua franca" : observations sur un mode de majoration », Cahiers de sociolinguistique, 2005/1 ( $\left.n^{\circ} 10\right)$, 167-178. Rennes : P.U.R. 
Vargas, C. (2009). « Peut-on inventer une grammaire pour la réussite scolaire? », Repères [En ligne], 39 | 2009, mis en ligne le 01 juin 2011, consulté le 15 février 2018. URL : http:// journals.openedition.org/reperes/365 ; DOI : 10.4000/reperes.365

Vargas, C. (2014). Les grammaires scolaires. De la recomposition à la reconfiguration. Aix en Provence : Presses universitaires de Provence.

Walker, J. (2010). « Norm-blindness and its effects on utterer-centred practice », Anglophonia, 14 (28) English Linguistics, 135-150. Toulouse : P.U.M.

Weber, C. (2004). « La culture grammaticale ordinaire : étude de verbalisations métagrammaticales et métacognitives d'apprenants natifs ", Langages 2004/2 ( $\left.{ }^{\circ} 154\right), 101-112$.

Yaguello, M. (1988). Catalogue des idées reçues sur la langue. Paris : Editions du Seuil.

\section{NOTES}

1. On utilise ici la dénomination "anglais langue seconde » par opposition à " anglais langue maternelle ou première ». Le propos n'est pas d'entrer dans la distinction entre ESL - « English as a Second Language » (sous toutes ses formes) et EFL/LVE, «English as a Foreign Language »/ Anglais Langue Vivante Étrangère.

2. comme dirait Cédric Villany.

3. on n'entrera pas ici dans la distinction franco-française entre « linguistique » et «sciences du langage ».

4. B. Hubert (2016: 118-119), formateur de professeurs de français à l'Université de Nantes, a recueilli des données auprès d'étudiants de Master 1, qui se destinent au Professorat des Ecoles. Ces données « mettent en évidence un rapport difficile avec la discipline « grammaire » (85\% des réponses), les formulations utilisées extraites du corpus sont à cet égard éloquentes : « répétitif ", « rébarbatif ", « fastidieux ", " pas facile ", « compliqué à comprendre ", « mauvais souvenir »...(..) Ce qui domine est un sentiment de non maîtrise ( «j'ai beaucoup de mal avec la matière ») ».

5. « Il existe dans notre société un véritable terrorisme de la grammaire » Yaguello (1988: 76 ).

6. Et déjà du temps de Molière, (Les Femmes savantes, VI, 2), Philaminte prône « La grammaire qui sait régenter jusqu'aux Rois,/Et les fait la main haute obéir à ses lois (...) »; et Bélise en prend la défense : « La grammaire, du verbe et du nominatif,/Comme de l'adjectif avec le substantif, /Nous enseigne les lois. », là où Martine (sa servante) ne voit pas l'intérêt de ce « jargon ».

7. Ainsi N. Kosciusko-Morizet: «Pour prolonger la réforme du primaire de 2008, je propose que les deux premières années du primaire soient consacrées à la consolidation des fondamentaux.(... ) Il faut mettre l'accent sur la grammaire, le vocabulaire, la capacité à exprimer, y compris oralement, une émotion, une idée, à argumenter. » La Croix,19 mai 2015.

8. Le Lievre (2008: 255-256).

9. «L'Anglais en France, une langue multiple: pratiques, représentations et postures d'apprentissage : une étude en contexte universitaire ».

10. Les spécialistes sont des anglicistes qui étudient 3 langues (voire une $4^{\text {ème}}$ ) dans l'Institut de perfectionnement en langues vivantes.

11. Le Lievre entend par là les étudiants non-spécialistes en langue, aussi appelés spécialistes d'autres disciplines (étudiants de LANSAD), qui étudient dans les Instituts de mathématiques appliquées, biologie appliquée et sciences de l'éducation, et suivent des cours de langue transversaux.

12. On ne peut s'empêcher de penser aux «Grammar schools » anglaises. Créées au $17^{\text {ème }}$ siècle pour permettre l'accès au savoir de tous les garçons, et non plus des seules classes sociales 
privilégiées, mais qui ont conservé cette appellation, issue du Moyen-Age, où l'on s'instruisait en apprenant la grammaire latine. A nouveau très sélectives aujourd'hui, en Angleterre !

13. voir Souesme (1989) et Granger (2001).

14. voir Birner (2015).

15. C'est B. Moscovici qui a proposé le concept de représentations sociales, en lien avec les travaux de Durkheim.

16. " Most people who did grammar in school have bad memories of it. They recall a dry, boring, irrelevant, learn-by-heart set of intellectual exercises. ». Partant de ce constat, Crystal invite à redécouvrir une grammaire, centrée sur la sémantique et à la pragmatique. Et il espère pouvoir changer le regard des « grammarphobes » : «It's not like that now. Or, at least, it doesn't have to be. »

17. « la question de l'enseignement de la grammaire, plus encore que celui d'autres domaines du français, met en échec les jeunes enseignants comme les enseignants confirmés (les tuteurs rechignent souvent à réaliser, devant leurs stagiaires, des séances centrées sur l'étude de la langue)».

18. Il faut ici affiner l'analyse et distinguer entre les représentations des élèves et celles des enseignants (vis à vis de leurs élèves, d'eux-mêmes ou de l'inspection académique, telles qu'elles s'expriment librement sur certains blogs).

19. « la grammaire est une matière confuse qui ne se "réfléchit " pas, où les réponses sont en vrai- faux » Hubert (2016:117)

20. «il existe deux types de grammaire: 1. la grammaire immanente, inhérente à la langue, c'est-à-dire les régularités que l'on peut observer dans la langue, et 2. la grammaire en tant que codification de règles, une grammaire codifiée par les grammairiens, la grammaire scolaire ou académique. La grammaire est donc inévitable, car elle fait partie de la langue; elle est immanente ; parler, c'est faire usage de la grammaire. La grammaire en tant que codification est, elle, évitable et parfois évitée car redoutable et redoutée. » Rotgé (2014 : 43)

21. On ne fait pas ici état d'un recueil scientifique de données, dans le cadre d'un protocole défini, mais de l'expression spontanée de demandes formulées sur la messagerie électronique de l'université, dans le cadre des cours d'agrégation, ou de Licence et de Master. Ce recueil a toutes ses limites, mais présente l'avantage de ne pas avoir été conditionné par des questions, dans le cadre d'un questionnaire ou d'interviews (à portée quantitative ou qualitative).

22. contrairement aux sciences exactes - mathématique, physique, chimie - qui utilisent couramment un langage qui leur est propre et les symboles logiques qui vont avec: $=, / /,-,+,>,<$, $\mathrm{W}, \Omega, \mathrm{A}, \mathrm{U}, \mathrm{I}, \mathrm{Na}+, \mathrm{NaCl}$ etc.

23. selon la racine grecque 'méta'.

24. J. Bouscaren dénonce «l'illusion (qui) consiste (...) à utiliser de façon quasi-fanatique des termes soi-disant consacrés », et " des enseignants qui ont la sensation regrettable qu'on doit impérativement utiliser tel terme à l'exclusion de tout autre. » Persec/Burgué $(2015,3)$.

25. On attire ici l'attention sur le fait que parler de «clarté » de la métalangue grammaticale ne rime pas forcément avec «simplicité ». On rejoint là-dessus Saussure (2002 : 236) : «Il n'y a pas du tout d'expression simple pour les choses à distinguer primairement en linguistique ; il ne peut pas y en avoir. " Sans doute parce que la linguistique est une science, qui décrit les langues à l'aide de concepts parfois très abstraits. Pour une meilleure compréhension des phénomènes, il faut, selon nous, commencer par réviser la terminologie, en prenant en compte de façon cohérente les trois plans de la syntaxe, de la sémantique et de la pragmatique. Cela permettra de redonner une vision juste des réalités grammaticales, et d'améliorer leur acquisition.

26. Pennec \& Delorme (2015, 34 et 180).

27. Dans l'enseignement supérieur en France, en L1, L2 et L3, y compris en classes préparatoires, l'enseignement de la grammaire est dispensé majoritairement en français, à l'image des manuels publiés. On note quelques exceptions, dont le Département d'Etudes du Monde Anglophone de 
l'Université Lumière Lyon2, où tous les cours de linguistique en L, dont la grammaire, sont intégralement dispensés en anglais. Pour l'enseignement secondaire, en collège et lycée, les manuels font apparaître la rubrique "grammaire » en français, ou parfois avec des titres en anglais et l'explication qui suit en français - on peut citer pour exemple les manuels New Password English $2^{\text {nde }}$ "Grammaire et Prononciation; Lexique »; et New Meeting Point Anglais 2de, «Language at work; improve your vocabulary and pronunciation; improve your speaking skills», puis questions traitées en français : "structures causatives, hypothèse, le style indirect, degrés de probabilité ; réagir et exprimer des sentiments, mots porteurs de sens, ton et intonation, savoir débattre ».

28. «Invariable, [le gérondif] est le plus souvent construit en français avec la préposition en (ou encore tout en); le complément d'une préposition étant toujours nominal, V-ant est alors la résultante d'une opération de nominalisation. (...) L'utilisation de ce mot en grammaire anglaise est sujette à caution, tous les linguistes ne lui accordant pas la même extension. Certains considèrent V-ing comme une nominalisation, d'autres lui attribuent des propriétés verbales. » Quivy (2015 : 293).

29. De là à décréter de ce fait que c'est une langue «facile », il n'y a qu'un pas. Or, si la difficulté d'une langue était liée aux déclinaisons, pourquoi tous les enfants arrivent-ils à parler dans leur langue natale, y compris pour les plus « complexes » sur ce plan-là ? (ex : le finnois, le polonais... ). C'est oublier aussi que toutes les langues ne fonctionnent pas sur le schéma de l'indo-européen, mais qu'il existe d'autres schémas comme les langues à tons (chinois mandarin), ou les langues à clic (zoulou, xhosa).

30. L'accent britannique du sud auquel on associe la Received Pronunciation, dite RP et qui correspond dans les esprits à l'anglais de la BBC (ou the Queen's English)

31. Le terme "variété » est à comprendre ici au sens sociolinguistique, comme un terme neutre qui désigne une forme de langue avec son système et sa cohérence propres, et qui diffère suffisamment des autres pour pouvoir s'en différencier.

32. Interview de Betty Birner, Professeur de Linguistique et Sciences Cognitives à Université de Northern Illinois. (2015).

33. On utilise ici le mot «communication » dans un sens très large, et sans prendre parti sur l'utilisation de l'anglais comme langue véhiculaire de communication, notamment dans l'éducation. On signale à ce sujet l'analyse très intéressante de Truchot (2005).

34. Corpus of Global Web-based English.

35. Culioli, A. (1986).

36. Dans sa Théorie des Opérations Énonciatives, Culioli lie les concepts de stabilité et d'ajustement. «Il me paraît utile d'insister sur le concept de stabilité. Sans stabilité, il n'y aurait pas d'ajustement régulier, pas de communication [...] mais la stabilité ne saurait être confondue avec la rigidité ou l'immuabilité. Les phénomènes linguistiques forment des systèmes dynamiques qui sont réguliers, mais avec une marge de variation due à des facteurs d'une grande diversité : on a affaire à des phénomènes qui sont à la fois stables et plastiques. [...] La déformation est une transformation qui modifie une configuration, de sorte que certaines propriétés restent invariantes sous transformation, tandis que d'autres vont varier. [...] Pour qu'il y ait déformabilité, il faut que l'on ait affaire à une forme schématique (telle qu'il puisse y avoir à la fois modification et invariance), que l'on ait des facteurs de déformation et que l'on ait une marge de jeu, un espace d'ajustement muni de propriétés topologiques.» (Culioli 1986, $\mathrm{T} 1: 129-130)$.

37. Boisson (2005 : 20-21) souligne «la respectabilité intellectuelle du niveau intermédiaire à forte orientation empirique, celui des lois, règles, contraintes, principes, modèles. " (niveau intermédiaire entre la saisie de 'faits' et la construction d'une théorie).

38. «Pour prendre un exemple caricatural, certains lecteurs anglophones doivent expliquer que le « $\mathrm{r} »$ dans les variétés non-rhotiques ne se prononce pas en position postvocalique quand eux- 
mêmes le prononcent spontanément. Plus généralement, leur système vocalique n'est pas rigoureusement celui de la variété de référence. » (Ballier, 2004 : §4)

39. Dans son acception large, $\mathrm{cf}$. note $\mathrm{n}^{\circ} 1$

40. Chevallard (1985)

41. A propos de la grammaire «scolaire ", Vargas (2009 et 2014) montre qu'elle procède plus par «recomposition» que par «transposition» de savoirs savants, en juxtaposant des savoirs empruntés à la linguistique et d'autres conservés de "la grammaire déjà-là » qui forment un «patchwork». L'auteur appelle à une véritable "reconfiguration» didactique des savoirs grammaticaux, qui fasse de la grammaire un objet cohérent, tant au niveau de l'analyse phrastique que textuelle, ou énonciative.

42. Dans une approche contrastive entre l'anglais et le français, et à l'image des études menées avec des étudiants par Lapaire (2016) pour l'anglais, et des élèves par Elalouf (2015) pour le français, on pourrait prendre, par exemple, comme objet d'étude « Time \& Tense » et s'interroger avec les étudiants sur la catégorisation des temps et de l'aspect (lien entre lexique et syntaxe), sur la morpho-syntaxe (lien entre régularité et irrégularité des formes, et pertinence du concept de «conjugaison»), sur le rôle et les caractéristiques d'un "auxiliaire», sur la singularité de «DO », ou des modaux, marqueurs prototypiques de l'anglais. Dans le secondaire, cela serait également exploitable pour les diverses notions et thématiques, du cycle 3 au cycle terminal, au travers de l'expression des repérages chronologiques temporels (vers le passé, le présent ou l'avenir) ou fictifs. D'autres pistes pourraient être suggérées.

43. Pour les apprenants-élèves, le cadre de l'approche actionnelle, à l'heure actuelle, ne précise et ne laisse pas vraiment de place pour l'enseignement la grammaire. Cf I.O. arrêté du 9-11-2015 J.O. du 24-11-2015 : dans cette présentation du cycle 3, qui inclut le Collège avec la classe de $6^{\text {ème }}$, la grammaire, dans l'enseignement des langues étrangères, est essentiellement liée aux objectifs culturels et communicationnels. Il faudrait proposer un enseignement explicite progressif de la grammaire, avec des phases de réflexivité (PRL) sur la langue anglaise, et amener les apprenantsélèves à saisir la cohérence de ce système linguistique qui donne accès au sens et le construit.

44. Cela concerne donc aussi les étudiants en formation de Licence, particulièrement pour ceux qui se destinent à l'enseignement et au MEEF. Cela paraît d'autant plus pertinent à ce niveau de formation, qu'un récent Référé de la Cour des Comptes (12 mars 2018) préconise de «placer les épreuves d'admissibilité [aux concours de recrutement pour l'enseignement] en fin de Licence » et la réponse des Ministres de l'Education Nationale et de l'Enseignement Supérieur (22 mai 2018) va dans le sens de cette réforme des ESPÉ et de la formation des enseignants.

https://www.ccomptes.fr/fr/publications/la-mise-en-place-des-ecoles-superieures-duprofessorat-et-de-leducation.

45. On pense par exemple aux recherches sur l'interaction du laboratoire ICAR (Université Lumière Lyon2- ENS Lyon), à l'enseignement et l'évaluation numériques de la grammaire anglaise à l'aide du logiciel TACIT-Grammar à l'université de Rennes, enfin à l'expérimentation de «Gamification du présentiel » dans des TDs de grammaire anglaise, à l'université de Paris 3.

\section{RÉSUMÉS}

Cet article s'intéresse au rapport que la grammaire entretient avec la langue, la métalangue et la linguistique, en prenant le cas de l'enseignement de l'anglais langue seconde. Il tente de comprendre d'où vient ce sentiment de non-maîtrise que le mot "grammaire », parfois redouté, 
suscite chez les apprenants (élèves ou étudiants, les étudiants stagiaires MEEF et même parfois les enseignants de l'anglais langue seconde). Dès lors, quelle grammaire enseigner et quels critères retenir pour enseigner efficacement la grammaire de l'anglais? L'analyse met à jour l'existence d'un double paradoxe, propre à la grammaire en général, et spécifique à la grammaire anglaise, et émet l'hypothèse que ces paradoxes sont liés à la fois aux représentations de la grammaire et à l'usage de la métalangue. Elle avance que le véritable enjeu de la grammaire, et de son enseignement, est de définir les contours de la grammaticalité. Si celle-ci s'évalue à l'épreuve de la langue, a-t-on besoin de "règles" grammaticales? L'article montre que la grammaire linguistique de l'anglais s'inscrit dans la tension entre ces deux exigences. Il propose pour conclure quelques pistes concrètes pour promouvoir l'enseignement de la grammaire de l'anglais, langue seconde, de manière fructueuse et efficace.

The study focuses on the relationships between grammar and language, as well as metalanguage and linguistics, taking the case of English as a foreign/second language. It seeks to understand why EFL/ESL students often have the feeling that they don't master grammar and why the very word "grammar" triggers fearful responses in students and sometimes even teachers. Consequently, what grammar should be taught and what criteria should be used to teach grammar effectively? The study addresses a double paradox that grammar and more specifically English grammar presents and shows that it has to do both with the representations of grammar and the use of metalanguage. What is at stake with grammar and grammar teaching is grammaticality. If this can only be assessed in the perspective of language use, are grammatical rules still necessary? The study shows that a linguistic grammar of English fits into these two competing requirements. It concludes by pointing out a few concrete ways to promote and teach English grammar effectively and efficiently.

\section{INDEX}

Mots-clés : grammaire linguistique, représentations, métalangue, terminologie grammaticale, règles, type, norme, grammaticalité, didactique de la grammaire, énonciation.

Keywords : linguistic grammar, representations, metalanguage, grammatical terminology, rules, types, norms, grammaticality, grammar teaching, enunciation.

\section{AUTEUR}

\section{CLOTILDE CASTAGNÉ-VÉZIÈS}

Université Lumière Lyon 2

Faculté des Langues - Département d'Etudes du Mondes Anglophone- Laboratoire CRTT 